\title{
DISC1 and SLC12A2 interaction affects human hippocampal function and connectivity
}

\author{
Joseph H. Callicott, ${ }^{1}$ Emer L. Feighery, ${ }^{1}$ Venkata S. Mattay, ${ }^{1,2}$ Michael G. White, \\ Qiang Chen, ${ }^{1,2}$ David A.A. Baranger, ${ }^{1}$ Karen F. Berman, ${ }^{1}$ Bai Lu, ${ }^{3}$ \\ Hongjun Song, ${ }^{4}$ Guo-li Ming, ${ }^{4}$ and Daniel R. Weinberger ${ }^{1,2,5}$
}

\begin{abstract}
${ }^{1}$ Clinical Brain Disorders Branch, Division of Intramural Programs, National Institute of Mental Health (NIMH), NIH, Bethesda, Maryland, USA.
${ }^{2}$ The Lieber Institute for Brain Development, Rangos Building, Johns Hopkins Medical Campus, Baltimore, Maryland, USA. ${ }^{3}$ GlaxoSmithKline, R\&D China, Shanghai, China. ${ }^{4}$ Institute for Cell Engineering, Departments of Neurology and Neuroscience, Johns Hopkins University School of Medicine, Baltimore, Maryland, USA. ${ }^{5}$ Departments of Psychiatry, Neurology, and Neuroscience and McKusick-Nathans Institute of Genetic Medicine, Johns Hopkins University School of Medicine, Baltimore, Maryland, USA.
\end{abstract}

\begin{abstract}
Hippocampal development is coordinated by both extracellular factors like GABA neurotransmission and intracellular components like DISC1. We previously reported that SLC12A2-dependent GABA depolarization and DISC1 coregulate hippocampal neuronal development, and 2 SNPs in these genes linked to mRNA expression interactively increase schizophrenia risk. Using functional MRI, we now confirm this biological interaction in vivo by showing in 2 independent samples of healthy individuals (total $N=349$ ) that subjects homozygous for both risk alleles evince dramatically decreased hippocampal area activation (Cohen's $d=0.78$ ) and connectivity $(d=0.57)$ during a recognition memory task. These data highlight the importance of epistatic models in understanding genetic association with complex brain phenotypes.
\end{abstract}

\section{Introduction}

Brain development is an emergent property of complex molecular interactions guiding cell growth and differentiation. As a well-studied example, hippocampal development appears to be dynamically regulated by both extrinsic and intrinsic mechanisms - GABA neurotransmission exemplifying the former and disrupted in schizophrenia 1 (DISC1) intracellular function exemplifying the latter. Kim et al. (1) recently addressed this possibility experimentally, reporting a significant interaction at both the molecular and the clinical level between SLC12A2 and DISC1. During adult and early postnatal hippocampal neurogenesis in the mouse, they showed that DISC1 knockdown-induced dendritic overgrowth of newborn neurons required GABA-induced depolarization, which is critically dependent on the abundant expression of SLC12A2. The effect of DISC1 knockdown was completely prevented by SLC12A2 knockdown. They also found a significant interaction between SNPs in DISC1 (rs1000731) and SLC12A2 (rs10089) and risk for schizophrenia in a combined analysis of 3 independent case-control samples. Subjects carrying minor alleles at both DISC1 rs1000731 and SLC12A2 rs10089 were at greater risk for schizophrenia compared with all other genotypes (combined analysis, $\mathrm{OR}=1.41, P=0.002$; likelihood ratio test, $P=0.0037)$, while neither SNP alone showed clinical association. As noted by Kim et al. (1), both SNPs were associated with gene expression effects in human brain, suggesting at least conceptually a clinical interaction echoing the molecular interaction in the basic animal model. Thus, based explicitly on these findings and using functional MRI (fMRI), we now report that healthy subjects carrying minor (i.e., risk) alleles in the same 2 SNPs show a significant decrease in hippocampal region activation and hippocampal connectivity with prefrontal cortex during a recognition memory task, confirming a biologic interaction between these genes on human hippocampal area function.

Conflict of interest: The authors have declared that no conflict of interest exists. Citation for this article: J Clin Invest. 2013;123(7):2961-2964. doi:10.1172/JCI67510.
GABA's inhibitory role in mature neurons depends on high levels of the chloride exporter SLC12A5 (also known as KCC2), whereas newborn neurons are depolarized by GABA due to high levels of the chloride importer SLC12A2 (also known as NKCC1) (2). During cortical development, the switch from SLC12A2 to SLC12A5 marks neuronal maturation in both hippocampus and prefrontal cortex and the SLC12A2/SLC12A5 ratio appears increased in schizophrenia, suggestive of an immature GABAergic neuronal phenotype (3). Abnormal levels of a key enzyme regulating SLC12A2 phosphorylation have also been described in schizophrenia (4). Moreover, evidence of reduced GABA function is a prominent finding in postmortem studies of schizophrenia brain tissue (5-7). Together, these data suggest that the molecular determinants of GABA's inhibitory influence are abnormal in schizophrenia.

DISC1 also has multiple roles in neuronal development including in growth, differentiation and migration, and synapse formation - that could negatively impact hippocampal area function and is a well-described potential risk gene for schizophrenia (8) that has been linked to abnormalities in hippocampal structure and function (9). First identified in a Scottish pedigree with a highly penetrant balanced translocation (1q42.1; 11q14.3) (10), DISC1 has common SNPs showing significant association in heterogeneous clinical samples via meta-analysis (SZGene, http:// www.szgene.org/; ref. 11). Located throughout the neuron with several unique protein-binding domains, DISC1 has a broad interactome, with potential reach into multiple cellular processes (12). Several studies have linked coding SNPs in DISC1 with alterations in brain structure and function in humans $(9,13)$ and with comparable structural abnormalities observed in transgenic DISC1 mice (14). Although genetic variations in SLC12A2 and DISC1 are not conclusively linked to schizophrenia, these data taken together suggest the potential for a combined negative impact by DISC1 and SLC12A2 on hippocampal function.

Thus, we hypothesized that the same DISC1 $\times$ SLC12A2 interaction would impact hippocampal area function in healthy adults, specifically hypothesizing that individuals carrying minor risk- 

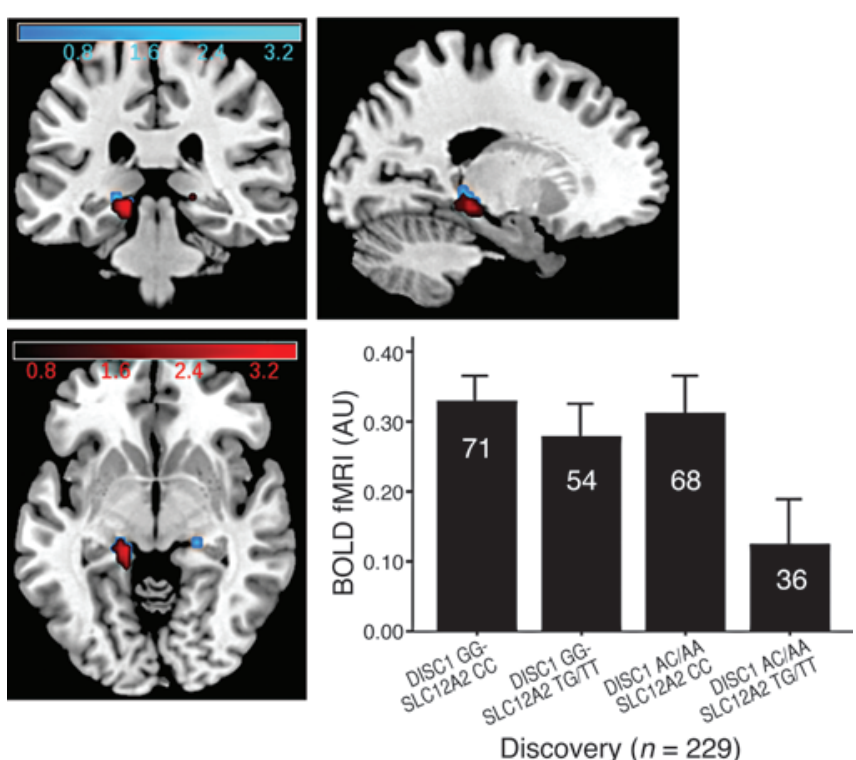

associated alleles in the same 2 SNPs in both genes would show altered hippocampal area function and coupling between the hippocampus and prefrontal cortex during a simple encoding memory paradigm (assayed by blood oxygen level-dependent [BOLD] fMRI) physiologic phenotypes associated with other putative schizophrenia risk genes $(9,15,16)$.

\section{Results and Discussion}

In our discovery fMRI cohort $(n=229)$, individuals with minor alleles at these same 2 SNPs had significantly reduced engagement of the left posterior hippocampal area (hippocampus and parahippocampal gyrus) compared with all other genotypes (Talairach coordinates $-14,-31,-1 ; P<0.05$ false discovery rate, corrected for small volume [FDR-SVC]) (ref. 17 and Figure 1, in red), none of which differed from each other. Connectivity was measured using a psychophysiological interaction (PPI) analysis (18) based on a seed placed within the left hippocampus proper (including anterior hippocampus). Minor allele homozygotes at both SNPs showed significantly reduced hippocampal to right ventrolateral prefrontal cortex (VLPFC) connectivity (Talairach coordinates 31, 21, 8; $P<0.05$ FDRSVC) (Figure 2, in red). There was also reduced left hippocampus to left VLPFC connectivity, which just missed significance after correction (Talairach coordinates $-44,36,7 ; P=0.06$ FDR-SVC).

We next explored this interaction in an independent healthy replication cohort collected and analyzed in an identical fashion $(n=120)$. We found the same significant interactions in precisely the same locations using regions of interest (ROIs) built from the discovery results. Power calculations for both activation (Cohen's $d=0.78)$ and connectivity $(d=0.57)$ based on the discovery cohort revealed moderate to large effect sizes and suggest that this replication sample was adequately powered for replication. We found reduced hippocampal engagement as expected on the left (Talairach coordinates $-11,-33,1 ; P<0.05$ FDR-SVC) within the discovery ROI (Talairach coordinates $-11,-33,-1 ; P<0.01$ uncorrected) (Figure 1 , in blue). However, we also found an area of reduced activation within the right anterior hippocampus (Talairach coordinates 22, $-3,-14 ; P<0.05$ FDR-SVC) in this sample. Using PPI in the replication sample, we again found reduced hippocampal to right VLPFC

\section{Figure 1}

DISC1 and SLC12A2 interaction negatively impacts hippocampal area function. Shown are BOLD signal changes in 2 cohorts of healthy subjects during a recognition memory task in the left posterior hippocampal region, with the discovery sample in red $(n=229)$ and the replication sample in blue $(n=120)$. In both, we found significantly decreased hippocampal area activation for individuals who are both DISC1 and SLC12A2 minor allele carriers $(P<0.05$ FDR for both). Replication results significantly and precisely fell within regions from the discovery sample based upon an ROI created from discovery results. Heat maps correspond to statistical $t$ values in BOLD signal activation and are displayed at $P<0.01$ uncorrected. The graph depicts parameter estimate extracted from the peak difference from the discovery sample (mean \pm SEM). Numbers in the graph bars indicate the number of subjects per group. connectivity (Talairach coordinates 49, 34, 3; $P<0.05$ FDR-SVC) within the discovery ROI (Talairach coordinates 49, 34, 6; $P<0.01$ uncorrected) (Figure 2, in blue). As before, we also found some support for reduced connectivity to left VLPFC (Talairach coordinates $-41,25,-10 ; P=0.05$ FDR-SVC), but this region was more inferior (extending to orbitofrontal cortex) and did not convincingly fall within an ROI based on the discovery left VLPFC connectivity findings (Talairach coordinates $-47,36,2 ; P=0.04$ uncorrected). Strikingly consistent with the results of the clinical association analyses (1), we did not find significant effects of either SNP individually in these cohorts, either in terms of hippocampal area engagement or connectivity, implicating the particular role for genetic interaction.

Neither of the 2 SNPs showing these significant interactions has been positive in large clinical genome-wide association studies for schizophrenia, but to date none of these studies have investigated gene $x$ gene interactions. It is the particular interaction in the absence of individual SNP effects, together with the molecular data from Kim et al. (1), which explicitly formed the hypotheses tested herein. Our results are consistent with the interpretation that the risk association of either of these genes is at least in part dependent on the other gene.

While the samples used here are relatively small by clinical association standards, they are relative large for imaging genetics studies, with a replication sample adequately powered according to discovery effect sizes. Our findings are consistent with other evidence that genetic associations that are weak at the level of clinical syndromes are much stronger at the level of brain physiology $(19,20)$. Replication across an independent cohort addresses some concerns about potential false-positive findings, but further replication is needed. Another potential concern is that the greatest genetic activation differences localized to posterior hippocampal areas and not in the dentate gyrus that was the focus of the molecular studies of Kim et al. (1). In the context of this particular memory task, which tends to show greatest activation in posterior hippocampus $(9,15,16)$, our findings within posterior hippocampal areas likely reflect multiple molecular events contributing to hippocampal development and processing, including within dentate gyrus. Thus, dysfunction within the anterior hip- 

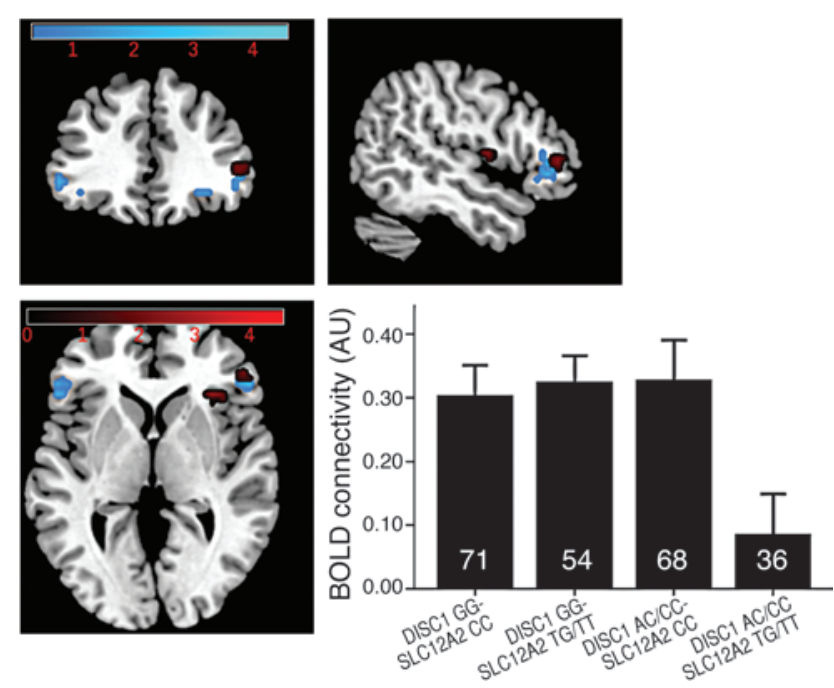

pocampus might affect hippocampal area function in general, but with greatest effect at some distance given the particular demands of the cognitive task. We found evidence for disruption within the anterior hippocampus in the replication sample. Also, connectivity findings were based on PPI analyses using a seed region placed explicitly within the hippocampus, which were at least suggestive of a more general abnormality in hippocampal area function. Nonetheless, the displacement into parahippocampal gyrus might be viewed as evidence that the molecular interaction in the developmental animal model (1) and the physiological interaction in adult humans represent different events.

While this latter possibility cannot be excluded here, it is important to note that fMRI does not have the spatial resolution to localize physiological activity in the dentate gyrus. The BOLD signals represent integrative field potentials over relatively large local networks spanning seconds rather than milliseconds. Standard fMRI analyses use normalization and smoothing that may shift further apparent activation. By involving complex visuals scenes, our task typically shows greatest activation in posterior hippocampal areas $(15,16)$. Thus, posterior locales had the strongest read out of the processing involved in the task, and it is the region known to integrate outflow from hippocampus to prefrontal cortex in memory processing. The reasons for stronger contralateral than ipsilateral connectivity findings are unclear; although at a liberal threshold we found reduced left hippocampus to left VLPFC connectivity in both cohorts. However, these contralateral connectivity findings echo prior reports using this same task (16) and the N-back working memory task and PET (21).

The broader ramifications of this interaction on hippocampal development and ongoing function and their implications for schizophrenia are beyond the reach of these imaging data. One may speculate that these findings suggest that variants without significant effects on their own interact to impair hippocampal area function and hippocampal connectivity and ultimately bias brain development on a path to schizophrenia (22), but further work directed specifically at clinical risk are necessary. Additionally, one can speculate that even minor impairment in hippocampal function or connectivity early in development will lead to an evolving cascade of broader developmental effects with impact on the emergence of illnesses like schizophrenia.

\section{Figure 2}

DISC1 and SLC12A2 interaction negatively impacts hippocampal connectivity. Using PPI using a seed within the left hippocampus, we found reduced connectivity with right VLPFC in both discovery (red, $n=229$ ) and replication (blue, $n=120$ ) samples for DISC1 and SLC12A2 minor allele carriers ( $P<0.05$ FDR for both). Replication results significantly and precisely fell within regions from the discovery sample based upon an $\mathrm{ROI}$ created from discovery results. Heat maps correspond to statistical $t$ values in connectivity and are displayed at $P<0.01$ uncorrected. The graph depicts parameter estimate extracted from the peak difference in connectivity from the discovery sample (mean \pm SEM). Numbers in the graph bars indicate the number of subjects per group.

The idea that genetic interactions may be important in illuminating pathology associated with disorders of complex heritability like schizophrenia is not new. Recently, Zuk and colleagues (23) argued that better accounting for gene-gene interactions is necessary to accurately estimate heritability and genetic risk. Genetic interactions have been shown to be critical in predicting effects of loss-of-function mutations in ion channel genes related to epilepsy (24) and are predominant in explaining quantitative traits in model organisms (25). Using functional neuroimaging, we have shown that such an interaction between an intrinsic biological factor (DISC1) and an extrinsic system (GABA signaling via SLC12A2) related to hippocampal neurogenesis in animal models and clinical risk for schizophrenia (1) has demonstrable effects on adult human hippocampal area function and hippocampal connectivity in vivo. Furthermore, these initial findings were replicated with remarkable fidelity in an independent sample. As in the clinical data upon which the specific SNP interaction was based, neither the DISC1 nor the SLC12A2 SNPs had significant independent effects on hippocampal area function or hippocampal connectivity. Our findings also illustrate that it is possible to translate molecular interactions related to basic brain developmental mechanisms into clinically relevant neurobiology and reiterate an important role for imaging genetics in reifying basic molecular interactions and clinical illness association in the context of human brain function.

\section{Methods}

We initially studied 229 healthy volunteers of mixed European descent between 18 and 60 years of age, who were recruited as part of the Clinical Brain Disorders Branch "Sibling Study" (9). We used standard methods to extract DNA from white blood cells and the TaqMan assay for genotyping (26). As previously described (1), we divided subjects into 4 groups that minimized minor allele carriers due to small sample size in some cells: major allele homozygotes (DISC1 GG-SLC12A2 CC; $n=71$ ), DISC1 major allele homozygotes plus SLC12A2 minor allele carriers (DISC1 GG-SLC12A2 CT/TT; $n=54$ ), SLC12A2 major allele homozygotes plus DISC1 A carriers (SLC12A2 CC-DISC1 GA/AA; $n=68$ ), and DISC1 and SLC12A2 CT/TT (DISC1 GA/ AA-SLC12A2 CT/TT carriers; $n=36$ ). The only significant demographic difference was age in the discovery sample, in which DISC1 GG-SLC12A2 CC and DISC1 GA/AA-SLC12A2 CT/TT groups were younger than the 
DISC1 GG-SLC12A2 CT/TT group $(P<0.05)$ (see Supplemental Table 1; supplemental material available online with this article; doi:10.1172/ JCI67510DS1). No subjects were taking psychotropic medications.

We collected whole brain BOLD fMRI data at 3T (9) using a recognition memory task (ref. 15; see Supplemental Methods for details). As expected with such a simple encoding task, there were no differences in accuracy or reaction time across genotypes. Data were analyzed in SPM5 (http://www.fil.ion. ucl.ac.uk/spm) (9), with individual contrasts (picture encoding versus visual fixation) modeling the genetic interaction as a random-effects, full factorial ANCOVA with covariates age and sex of no interest. Given our prior hypotheses, we restricted our analysis to bilateral hippocampal areas (hippocampus plus parahippocampal gyrus), with significance at $P<0.05$ FDR-SVC (17). We used PPI within SPM5 (18) to examine the connectivity between the hippocampus proper and VLPFC based on prior findings (ref. 16; see Supplemental Methods for details). We used identical contrasts and covariates for both BOLD activation and PPI analyses. Individual PPI contrasts were entered into a full factorial ANCOVA, covaried for age and sex with a statistical threshold of $P<0.05$ FDRSVC. Our results from discovery were used to create ROIs as defined by voxels surviving the corrected thresholds for activation and connectivity, respectively. These ROIs were used to query the replication sample.

Using the identical protocol, we then obtained AMRI data for 120 additional healthy subjects of mixed European descent ( 71 healthy volunteer subjects and 49 healthy and unrelated unaffected siblings of patients with schizophrenia) (see Supplemental Table 1). Only one sibling per family was included to maintain independence. Inclusion criteria were the same, including that unaffected siblings had neither a history of psychiatric illness nor medications. All data were collected, processed, and analyzed in an identical fashion to the discovery sample and divided into the same groups: DISC1 GG-SLC12A2 CC $(n=49)$, DISC1 GG-SLC12A2 CT/TT $(n=23)$, SLC12A2 CCDISC1 GA/AA $(n=33)$, and DISC1 GA/AA-SLC12A2 CT/TT carriers $(n=15)$. There were no significant demographic or task performance differences nor were there any differences in the ratio of healthy subjects-to-healthy siblings across genotype groups. As in the discovery cohort, we used identical contrasts and covariates for both BOLD activation and PPI analyses.
Statistics. In summary, all fMRI analyses were statistically analyzed within SPM5, while demographic differences were examined using SPSS. For demographic measures, we used ANOVA with genotype group as the main effect $(P<0.05)$ (see Supplemental Table 1). For all fMRI data, both whole brain activation and PPI, we used ANCOVAs within SPM5 that included age and sex as covariates of no interest. All analyses in both cohorts were repeated without these covariates, and we found no significant differences in any result. Reported results can be considered 2-sided $t$ tests, and fMRI results are specific to the interaction term in all cases. For discovery, we chose a statistical threshold for fMRI activation and connectivity results of $P<0.05$ FDR-SVC (17). We used the initial activation and connectivity results (separately) as ROIs for replication, with a statistical threshold of $P<0.01$ uncorrected. We extracted measures of BOLD fMRI activation and connectivity (parameter estimates) from significant clusters subsequently used to create graphs using SPSS. All graphs depict mean \pm SEM.

Study approval. All subjects were recruited as part of the Clinical Brain Disorders Branch "Sibling Study" (NCT00001486; ref. 9). This protocol was approved by the Institutional Review Board of the NIMH (Office of Human Subjects Research Protections). All subjects gave written, informed consent prior to participation.

\section{Acknowledgments}

Federal funding was from the NIMH Intramural Research Programs (to Daniel R. Weinberger). We would like to thank our patients and their families for participating in our research.

Received for publication October 24, 2012, and accepted in revised form April 11, 2013.

Address correspondence to: Joseph H. Callicott, Head, Unit on Dynamic Imaging Genetics, Clinical Brain Disorders Branch, DIRP, NIMH, NIH, 10 Center Drive, Room 3C-117, MSC 1379, Bethesda, Maryland 20892-1379, USA. Phone: 301.402.3018; Fax: 301.480.7795; E-mail: callicottj@mail.nih.gov.
1. Kim JY, et al. Interplay between DISC1 and GABA signaling regulates neurogenesis in mice and risk for schizophrenia. Cell. 2012;148(5):1051-1064.

2. Owens DF, Kreigstein AR. Is there more to GABA than synaptic inhibition? Nat Rev Neurosci. 2002; 3(9):7157-7127.

3. Hyde TM, et al. Expression of GABA signaling molecules KCC2, SLC12A2, and GAD1 in cortical development and schizophrenia. J Neurosci. 2011;31(30):11088-11095.

4. Arion D, Lewis DA. Altered expression of regulators of the cortical chloride transporters NKCC1 and KCC2 in schizophrenia. Arch Gen Psychiatry. 2011;68(1):21-31.

5. Lewis DA, Hashimoto T, Volk DW. Cortical inhibitory neurons and schizophrenia. Nat Rev Neurosci. 2005;6(4):312-324.

6. Lewis DA, Moghaddam B. Cognitive dysfunction in schizophrenia: convergence of gamma-aminobutyric acid and glutamate alterations. Arch Neurol. 2006;63(10):1372-1376.

7. Curley AA, Lewis DA. Cortical basket cell dysfunction in schizophrenia. J Physiol. 2012;590(pt 4):715-724.

8. Porteous DJ, Millar JK, Brandon NJ, Sawa A. DISC1 at 10: connecting psychiatric genetics and neuroscience. Trends Mol Med. 2011;17(12):699-706.

9. Callicott JH, et al. Variation in DISC1 affects hippocampal structure and function and increases risk for schizophrenia. Proc Natl Acad Sci U S A 2005;102(24):8627-8632.

10. St Clair D, et al. Association within a family of a balanced autosomal translocation with major mental illness. Lancet. 1990;336(8706):13-16.

11. Allen NC, et al. Systematic meta-analyses and field synopsis of genetic association studies in schizophrenia: the SzGene database. Nat Genet. 2008; 40(7):827-834.

12. Brandon NJ, Sawa A. Linking neurodevelopmental and synaptic theories of mental illness through DISC1. Nat Rev Neurosci. 2011;12(12):707-22.

13. Cannon TD, et al. Association of DISC1/TRAX haplotypes with schizophrenia, reduced prefrontal gray matter, and impaired short- and long-term memory. Arch Gen Psychiatry. 2005;62(11):1205-1213.

14. Hikida $T$, et al. Dominant-negative DISC1 transgenic mice display schizophrenia-associated phenotypes detected by measures translatable to humans. Proc Natl Acad Sci U S A. 2007; 104(36): 14501-14506.

15. Hariri AR, et al. Brain-derived neurotrophic factor val66met polymorphism affects human memory-related hippocampal activity and predicts memory performance. J Neurosci. 2003; 23(17):6690-6694

16. Bertolino A, et al. Prefrontal-hippocampal coupling during memory processing is modulated by COMT val158met genotype. Biol Psychiatry. 2006;60(11):1250-1258

17. Genovese CR, Lazar NA, Nichols T. Thresholding of statistical maps in functional neuroimaging using the false discovery rate. Neuroimage. 2002;15(4):870-878.

18. Friston KJ, Buechel C, Fink GR, Morris J, Rolls E, Dolan RJ. Psychophysiological and modula- tory interactions in neuroimaging. Neuroimage. 1997;6(3):218-229

19. Huffaker SJ, et al. A primate-specific, brain isoform of $\mathrm{KCNH} 2$ affects cortical physiology, cognition, neuronal repolarization and risk of schizophrenia. Nat Med. 2009;15(5):509-518.

20. Mier D, Kirsch P, Meyer-Lindenberg A. Neural substrates of pleiotropic action of genetic variation in COMT: a meta-analysis. Mol Psychiatry. 2010;15(9):918-927.

21. Meyer-Lindenberg AS, et al. Regionally specific disturbance of dorsolateral prefrontal-hippocampal functional connectivity in schizophrenia. Arch Gen Psychiatry. 2005;62(4):379-386.

22. Weinberger DR, Levitt P. Neurodevelopmental origins of schizophrenia. In: Weinberger DR, Harrison PJ, eds. Schizophrenia. 3rd ed. Oxford, United Kingdom: Wiley-Blackwell; 2011:393-412.

23. Zuk O, Hechtera E, Sunyaev SR, Lander ES. The mystery of missing heritability: Genetic interactions create phantom heritability. Proc Natl Acad Sci US A. 2012;109(4):1193-1198.

24. Klassen R, et al. Exome sequencing of ion channel genes reveals complex profiles confounding personal risk assessment in epilepsy. Cell. 2011;145(7):1036-1048

25. Huang W, et al. Epistasis dominates the genetic architecture of Drosophila quantitative traits. Proc Natl Acad Sci U S A. 2012;109(39):15553-15559.

26. Livak KJ. Allelic discrimination using fluorogenic probes and the $5^{\prime}$ nuclease assay. Genet Anal. 1999;14(5-6):143-149. 